\section{E-MRS Holds Summer School on Current Problems of Semiconductor Surfaces and Interfaces}

Fermi once said, "If a solid volume is a God, its surface is the Devil." This is no longer true since so many scientists have tackled the structure of solid surfaces with a large number of sophisticated instruments. Last summer in Bavaria, 80 scientists from Europe and the United States reviewed present knowledge on semiconductor interfaces and surfaces and their impact on semiconductor devices at a fiveday summer school.

A flavor of Cerman tradition enveloped the summer school on "Current Problems of Semiconductor Surfaces and Interfaces" held June 30 to July 4, 1986 in Burghausen, West Germany. The first dinner was served in the vaulted room where, in 1373, the duke of Bavaria sold his land of Brandenburg (which later became Prussia) for 250,000 guldens. Attendees could also view many Bavarian lakes and churches.

The school was hosted by WackerChemitronic, producer of almost $40 \%$ of the hyperpure silicon needed worldwide. Wacker-Chemitronic, the Council of Europe, and E-MRS were the principal funding organizations. The program, organized by $H$. Jacob and E. Sirtl (Wacker-Chemitronic and Heliotronic) and by $M$. Rodot and J. Derrien (CNRS), covered four main topics: fundamental aspects of semiconductor surfaces and interfaces, surface and interface technology and diagnostics, specific interfaces, and homoepitaxy of semiconductors.

Clean surfaces were described by $G$. Le Lay (CNRS, Marseille) and A. Kahn (Princeton University, New Jersey) for Si and III$V_{s}$, respectively. The grow th and structure of layers deposited on such surfaces, beginning with a small fraction of a monolayer, were discussed by J. Derrien (CNRS, Grenoble) and J.A. Venables (University of Sussex, Brighton). Several modern diagnostic techniques, together with examples of recent research results, were reviewed as follows: photoemission by $W$. Mönch (Duisburg University) and F.J. Himpsel (IBM); vibration spectroscopy by $\mathrm{H}$. Ibach (Institute of Nuclear Research, Jülich); LEED by $M$. Henzler (Hannover University), SIMS by A.M. Huber (Thomson-CSF).

Special mention is due to scanning tunneling microscopy, a new technique presented by R.J. Behm (Munich University). It has a very high potential value, as recognized by most specialists, to characterize the topography and electronic and chemical states of surfaces with a resolution of a few angstroms.

Schottky Barriers

Schottky barriers were one of the

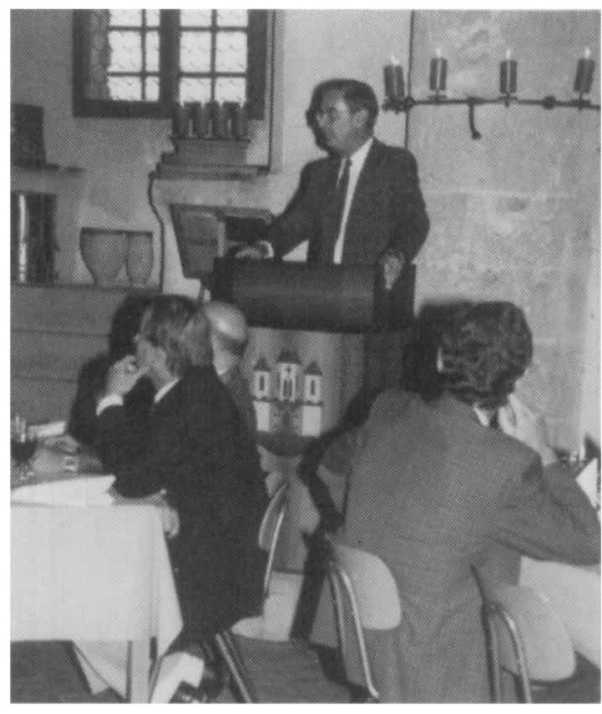

W. Freiesleben, president of WackerChemitronic and Wacker-Siltronic Companies, welcomes attendees.

meeting's highlights. It is difficult to believe that, half a century after their first description, we are not able to account for the observed barrier heights. While it is known that some "interface states" control this feature, the origin of these states is controversial. The choice is between MIGS (metal-induced gap states), which were pledged by F. Flores (Madrid University), and states due to defects inside the semiconductor. L. Brillson (Xerox) and A.B. McLean (Cardiff University) showed how microscopic interface modifications (including chemical reactions) may modify Schottky barrier heights in a way that pleads for defect states rather than MIGS. Since interface states do exist, they should be made measurable: that task was fulfilled by $C$. Barret (Orsay University) by transport measurements at variable temperatures and frequencies, adding further credibility to the model of defect states.

\section{Industry Practices and Needs}

Many presentations started from the practice and needs of the microelectronics industry: wafer surface preparation (D. Huber, Wacker-Chemitronic, and J. B. Theeten, LEP); chemical vapor deposition (M. Druminski, Siemens, and M.W.M. Graef, Philips); molecular beam epitaxy of Si (E. Kasper, AEG, and F. Arnaud d'Avitaya, CNET Grenoble). For instance, MBE specialists established that steep doping of layers was made easier by applying an electric field or impinging an electronic beam onto the growing layer. $M B E$ is often applied to III-V microstructures, whose electronic properties and applications were reviewed by H.R. Rupprecht (Fraunhofer Institute, Freiburg) and K. Ploog (Max Planck Institute, Stuttgart). The control and reliability of very thin $(10 \mathrm{~nm})$, high quality oxide layers were discussed by R.F. de Keersmaecker (IMEC, Leuven) and D.R. Wolters
(Philips), while the metal-polyimide bond was described by P. Ho (IBM).

Attendees also heard about other key technological problems, such as the gettering techniques capable of increasing crystal perfection (B.O. Kolbesen, Siemens), and metallic contacts, dealt with in three papers. F. Jahnel (Siemens) explained the difficulties frequently met in several types of metallization used in VLSI; for instance, the Ta-Si contact s could suffer. consumption, cancer, pocks, measles, cellulitis obscura, and more. G. Bomchil's (CNET) papercentered on the formation of refractory silicides, and M.A. Nicolet's (California Institute of Technology) on diffusion barriers (e:g. TiN) which may become necessary in some cases. Finally, H.T.G. Hentzell (Linköping University), studying the influence of impurities on the recrystallization of amorphous $\mathrm{Si}$, created a surprise by finding traces of a $\mathrm{Al}$ silicide as an intermediate step in this process.

Research is so active in this field that numerous opportunities for debate are being prepared. One of these will be the Second International Conference on the Formation of Semiconductor Interfaces, to be held in 1988 in Osaka (Japan) under the direction of A. Hiraki.

M. RODOT

\section{E-MRS Symposium Features Advanced Materials for Telecommunication}

Symposium $A$ at the E-MRS Spring Meeting held in Strasbourg, France during the week of June 16, 1986 featured eight sessions on "Advanced Materials for Telecommunication."

The symposium organizers were P.A. Glasow (Siemens AC Research Laboratories, West Germany); Y.I. Nissim and J.P. Noblanc (CNET, France); and J. Speight (British Telecom Research, Great Britain).

A recognition that progress in telecommunication is intimately tied to advances in electronic materials prompted this fourday symposium concerned with all aspects of materials for modern and future telecommunication. The complete materials aspect-from emitters through transmitters and receivers-was covered. The symposium included the wide range of bulk III-V materials growth, all aspects of semiconductor materials analysis, and correlations of bulk material-processing-device properties. The symposium covered the various kinds of semiconductor heterostructures and their characterization. Materials for optoelectronic integration were also addressed as were contacts, interconnects, insulation, and passivation.

The symposium's eight sessions featured 28 invited papers and 48 contributed papers

Continued 